\title{
HEAT TRANSFER BETWEEN A FLUID AND A PLATE: MULTIDIMENSIONAL LAPLACE TRANSFORMATION METHODS
}

\author{
R.G. BUSCHMAN \\ Department of Mathematics \\ University of Wyoming \\ Box 3036, University Station \\ Laramie, WY 82071, U.S.A. \\ (Recelved February 4, 1982)
}

\begin{abstract}
Multidimensional Laplace transformations are used to obtain the surface temperature and the surface heat flux of a plate with a fluid flowing across it without solving the complete boundary value problem. It 18 also shown that the constant initial and boundary values can be relaxed and the method still applies. The solution to the boundary value problem at points away from the surface can be treated similarly.
\end{abstract}

KEY WORDS AND PHRASES. Multidimensional Laplace transformations, boundary value problems, heat transfer, compatability conditions, erron functions. AMS SUBJECT CLASSIFICATION (1979). Primary 44A30, Secondary 35K20, 80A20, 44A10, $33420,44 A 20$.

1. INTRODUCTION.

Recently James Sucec $[1,2]$ considered a problem which Involves heat transfer between a plate and a fluid which is flowing across the plate. Th1s work was extended by P. Singh, V. P. Sharma, and U. N. Misra [3] to the case of a porous plate with suction. In both cases it is assumed that constant property, laminar, slug flow occurs across the plate and that the plate 18 convectively cooled from below. The Laplace transformation techniques are used in order to obtain the temperature function at points within the fluid and, from this, the surface heat flux and temperature of the plate is obtained. The work is in the spirit of 
Carslaw and Jaeger [4] In finding "exact" or "analytic" solutions. Several related problems and the detalls of the mathematical model are discussed with care in $[1,2]$. In this paper we show that the direct application of the multidimensional Laplace transformation to the boundary value problem, with the assumption of the existence of the transform of the solution as a replacement for the bounded solution condition, leads directly to a "compatability" condition. The transforms of the surface heat flux and temperature can then be computed directly by use of this compatability condition along with the transform of the boundary condition, without first obtaining the temperature function at points within the fluid. These transforms are then inverted. Some simpler applications of a compatability condition were presented by $D$. Voelker and G. Doetsch [5] a number of years ago. In Sections 2 and 3 we utilize this method to obtain the results of Sucec $[1,2]$ and of Singh, Sharma, and Misra [3], respectively. In the last section we show how the method can be applied as well to similar problems in which arbitrary functions are introduced into the initial conditions and the boundary conditions.

We use reference notations to tabulated pairs of functions which are related by the Laplace transformation such as $[2: 2.1(22)]^{-1}$ to denote Formula 22 , Section 2.1 of the inversion part of [2], or [6:A4(5)] to denote Formula 5 of Section $A 4$ of [6]. A few useful combinations from tables are recorded in our Appendix.

2. THE PROBLEM OF SUCEC.

In order to simplify the boundary value problem of Sucec $[1,2]$, we rescale the variables and introduce some new constants:

$$
\begin{aligned}
& \theta=\alpha_{f} r \theta_{1}+\theta_{c}, \quad t=\alpha_{f} r^{2} t_{1}, \quad x=\alpha_{f} r^{2} u_{\infty} x_{1}, \\
& y=\alpha_{f} r y_{1}, \quad \beta=\alpha_{f} r h_{c} / k_{f}, \quad r=-\theta_{c} /\left(\alpha_{f} r\right) .
\end{aligned}
$$

If we subsequently drop the subscripts, the boundary value problem can be rewritten In the form

$$
\begin{aligned}
& \theta_{t}(t, x, y)+\theta_{x}(t, x, y)=\theta_{y y}(t, x, y), \\
& \theta(0+, x, y)=0, \\
& \theta(t, 0+, y)=\gamma,
\end{aligned}
$$




$$
\theta_{y}(t, x, 0+)=B \theta(t, x, 0+)+\theta_{t}(t, x, 0+) .
$$

We also replace the condition that $\theta(t, x, y)$ be bounded, by the assumption that the solution possesses a 3-dimensional Laplace transform, that is

$$
c^{3}\{\theta(t, x, y)\}=f(s, u, v)
$$

Properties of the 2-dimensional Laplace transformation are developed in [5], wich further Includes an extensive inversion table. Initially we transform with respect to the first two variables and let

$$
L^{2}\{\theta(t, x, y)\}=8(8, u, y)
$$

If we next transform with respect to the third variable, then, after some simplificat1ons, the problem becomes

$$
\begin{gathered}
f(s, u, v)=\frac{(v+8+\beta) g(x, u, 0+)-\gamma(8 v)^{-1}}{v^{2}-(u+8)}, \\
g_{y}(8, u, 0+)=(8+\beta) g(8, u, 0+) .
\end{gathered}
$$

In (2.8) we observe that the denominator is zero arbitrar1ly far out in right half planes; thus, f 18 not analytic In right half planes, unless the numerator 18 also zero for $v=(u+8)^{1 / 2}$. Hence, $f$ cannot be a Laplace transform in general. The application of the condition for existence (2.6) leads us to the "compatab1lity cond1t1on"

$$
\left((u+8)^{1 / 2}+8+\beta\right) g(8, u, 0+)-\gamma^{-1}(u+8)^{-1 / 2}=0
$$

This condition (2.10) can be used in order to eliminate the, as yet undetermined, function $g(s, u, 0+)$ from (2.8). Thus f can be expressed as a Laplace transform which can then be Inverted by the use of tables. We further note that the Inversion of (2.10) 1tself leads to the temperature at the boundary. Further, the elimination of $8(8, u, 0+)$ between $(2.9)$ and $(2.10)$ and the Inversion of the result leads to the heat flux at the boundary. Hence both $\theta(t, x, 0+)$ and $\theta_{y}(t, x, 0+)$ can be obtained without, and In an easier manner than, going through the Inversion of $f(s, u, v)$ in order to first obtain $\theta(t, x, y)$.

For the Inversions of (2.9) and (2.10), we need the 2-dimensional Inversions of 


$$
\begin{gathered}
g_{y}(x, u, 0+)=\gamma(s+\beta) s^{-1} w^{-1 / 2}\left(w^{1 / 2}+8+\beta\right)^{-1}, \\
g(s, u, 0+)=\gamma s^{-1} w^{-1 / 2}\left(w^{1 / 2}+8+\beta\right)^{-1},
\end{gathered}
$$

In which $w=8+u$. The linear substitution formula $[6: \wedge 4(5)]$ is designed to help with such situations. If we apply it to (A.1) from our Appendix, we have

$$
\theta(t, x, 0+)=\gamma e^{\beta^{2} x}\left(\operatorname{Erfc}\left(B x^{1 / 2}\right)-\operatorname{Brfc}\left(B x^{1 / 2}+\frac{t-x}{2 x^{1 / 2}}\right)\right) U(t-x) ;
$$

if further we use $[6: B 2(13)]$, we have

$$
\theta_{y}(t, x, 0+)=\beta \theta(t, x, 0+)+\gamma e^{-\beta(t-x)} x(t-x, x) U(t-x) .
$$

In these last two formulas, the unit step function 18 denoted by $U$ and a heat kernel by

$$
x(a, b)=(\pi b)^{-1 / 2} e^{-a^{2} /(4 b)} .
$$

The elimination of $g(8, u, 0+)$ from $(2.8)$ and $(2.10)$, followed by laversion, In which we use the same type of results as for (2.13), along with (A.2), leads to

$$
\begin{aligned}
\theta(t, x, y) & =\gamma U(t-x)\left\{1-\operatorname{Erfc}\left(\frac{y}{2 x^{1 / 2}}\right)+\right. \\
& \left.+e^{\beta^{2} x+B y}\left(\operatorname{Erfc}\left(\frac{y}{2 x^{1 / 2}}+B x^{1 / 2}\right)-\operatorname{Erfc}\left(\frac{t-x+y}{2 x^{1 / 2}}+B x^{1 / 2}\right)\right)\right\} .
\end{aligned}
$$

The results (2.13), (2.14), and (2.16) correspond to those of Sucec $[1,2]$.

3. THE PROBLEM OF SINGH, SHARMA, AND MISRA.

If we use the same rescaling and further let $2 \mu=v_{0} r$, the problem can be written in the form

$$
\begin{aligned}
& \theta_{t}(t, x, y)+\theta_{x}(t, x, y)+2 \mu \theta_{y}(t, x, y)=\theta_{y y}(t, x, y), \\
& \theta(0+, x, y)=0, \\
& \theta(t, 0+, y)=\gamma \\
& \theta_{y}(t, x, 0+)=\beta \theta(t, x, 0+)+\theta_{t}(t, x, 0+), \\
& f^{3}\{\theta(t, x, y)\}=f(s, u, v) \text { exists. }
\end{aligned}
$$

A completely analogous development leads to the compatability condition 


$$
\left(w^{1 / 2}+s+\beta-\mu\right) g(s, u, 0+)-\gamma s^{-1} \cdot\left(w^{1 / 2}+\mu\right)^{-1}=0,
$$

in which $w=u+s+\mu^{2}$. Because of the similarity to the forms of Section 2 , we can make use of analogous computations to obtain $\theta(t, x, 0+)$ and $\theta_{y}(t, x, 0+), a g a i n$ without obtaining $\theta(t, x, y)$. Hence,

$$
\begin{aligned}
\theta(t, x, 0+) & =\frac{\gamma}{\beta-2 \mu}\left\{(\beta-\mu) e^{\beta(\beta-2 \mu) \operatorname{xx}} \operatorname{Erfc}\left((\beta-\mu) x^{1 / 2}\right)-\operatorname{Erfc}\left(\frac{t-x}{2 x^{1 / 2}}+(\beta-\mu) x^{1 / 2}\right)\right)+ \\
& \left.+\mu e^{-(\beta-2 \mu)(t-x)} \operatorname{Erfc}\left(\frac{t-x}{2 x^{1 / 2}+\mu x^{1 / 2}}\right)-\mu \operatorname{Erfc}\left\{\mu x^{1 / 2}\right)\right\} U(t-x),
\end{aligned}
$$

and similarly

$$
\begin{aligned}
\theta_{y}(t, x, 0+) & =\beta \theta(t, x, 0+)+\left(\gamma e^{-(\beta-\mu)(t-x)-\mu^{2} x} x(t-x, x)-\right. \\
& \left.-\gamma \mu e^{(\beta-2 \mu)(t-x)} \operatorname{Erfc}\left(\frac{t-x}{2 x^{1 / 2}}+\mu x^{1 / 2}\right)\right) U(t-x),
\end{aligned}
$$

which agree with the results in [3]. The formula for $\theta(t, x, y)$ analogous to (2.16), can be obtalned:

$$
\begin{aligned}
& \theta(t, x, y)=\gamma\left\{1-\frac{1}{\beta-2 \mu}\left\{\frac{\beta}{2} e^{2 \mu y} \operatorname{Erfc}\left(\frac{y}{2 x^{1 / 2}}+\mu x^{1 / 2}\right)+\frac{\beta-2 \mu}{2} \operatorname{Erfc}\left(\frac{y}{2 x^{1 / 2}-\mu x^{1 / 2}}\right)+\right.\right. \\
& +(\beta-\mu) e^{\beta y+\beta(\beta-2 \mu) x}\left(\operatorname{Erfc}\left(\frac{y+t-x}{2 x^{1 / 2}}+(\beta-\mu) x^{1 / 2}\right)-\operatorname{Erfc}\left(\frac{y}{2 x^{1 / 2}}+(\beta-\mu) x^{1 / 2}\right)\right)- \\
& \left.\left.-\mu e^{2 \mu y-(\beta-2 \mu)(t-x)} \operatorname{Erfc}\left(\frac{y+t-x}{2 x^{1 / 2}+\mu x^{1 / 2}}\right)\right)\right\} U(t-x) .
\end{aligned}
$$

\section{GENERALIZATIONS.}

If we allow for other than a constant inftial temperature distribution through $A(x, y)$, for changes in inlet temperature by $B(t, y)$, and for changes in temperature due to the coolant by $c(t, x)$, the rescaled problem can be written in the general form

$$
\begin{aligned}
& \theta_{t}(t, x, y)+\theta_{x}(t, x, y)+2 \mu \theta_{y}(t, x, y)=\theta_{y y}(t, x, y), \\
& \theta(0+, x, y)=A(x, y), \\
& \theta(t, 0+, y)=B(t, y),
\end{aligned}
$$




$$
\begin{aligned}
& \theta_{y}(t, x, 0+)=B(\theta(t, x, 0+)-C(t, x))=\theta_{t}(t, x+), \\
& C^{3}\{\theta(t, x, y)\}=f(8, u, v) \text { exists. }
\end{aligned}
$$

The problem lacks symetry only in (4.4); $\mu=0$ provides the direct generalization of Sucec's problem.

The application of the Laplace transformations again, as in Sections 2 and 3 , lead to the consideration of the denominator in which $v-\mu=w^{1 / 2}, w=s+u+\mu^{2}$ Is now critical. Hence we must have the compatability condition

$$
\begin{gathered}
\left(w^{1 / 2}+s+B-\mu\right) g(8, u, 0+)=a\left(u, w^{1 / 2}+\mu\right)+ \\
+b\left(s, w^{1 / 2}+\mu\right)-\beta c(8, u)-a(u, 0+)
\end{gathered}
$$

for which we also need the continulty assumption $a(u, 0+)=c(0+, u)$. We have introduced the notations $c^{2}\{A(x, y)\}=a(u, v), c^{2}\{B(t, y)\}=b(s, v), c^{2}\{c(t, x)\}=c(s, u)$, and $C\{A(x, 0+)\}=a(u, 0+)$. After some calculations we obtain the transforms of the desired functions

$$
\begin{aligned}
& g(8, u, 0+)=\frac{a\left(u, w^{1 / 2}+\mu\right)+b\left(8, w^{1 / 2}+\mu\right)+\beta c(8, u)+a(u, 0+)}{w^{1 / 2}+8+\beta-\mu}, \\
& g_{y}(s, u, 0+)=\frac{(s+\beta)\left(a\left(u, w^{1 / 2}+\mu\right)\right)+b\left(s, w^{1 / 2}+\mu\right)}{w^{1 / 2}+s+\beta-\mu}- \\
& -\frac{\left(w^{1 / 2}-\mu\right)(\beta c(s, u)+a(u, 0+))}{w^{1 / 2}+s+\beta-\mu} \text {, } \\
& f(s, u, v)=-\frac{\left(w^{1 / 2}+\mu\right) a(u, v)-v a\left(u, w^{1 / 2}+\mu\right)}{\left(w^{1 / 2}+8+\beta-\mu\right)\left((v-\mu)^{2}-w\right)}-\frac{\left(w^{1 / 2}+\mu\right) b(8, v)-v b\left(8, w^{1 / 2}+\mu\right)}{\left(w^{1 / 2}+8+\beta-\mu\right)\left((v-\mu)^{2}-w\right)}- \\
& -\frac{(8+\beta-2 \mu)\left[a(u, v)-a\left(u, w^{1 / 2}+\mu\right)+b(8, v)-b\left(8, w^{1 / 2}+\mu\right)\right]}{\left(w^{1 / 2}+8+\beta-\mu\right)\left((v-\mu)^{2}-w\right)} \\
& +\frac{\beta c(x, u)+a(u, 0+)}{\left(w^{1 / 2}+\beta+\beta-\mu\right)\left(v-\mu+w^{1 / 2}\right)} .
\end{aligned}
$$

The terms of equation (4.9) are set up in forms simllar to the differences which appear in the tables [5]. Lengthy calculations involving convolution formulas, entries such as those in the Appendix, and extensions of entries from the tables [6] 
and [5] are needed to Invert these transforms, especially the terms of (4.9); the results involve various convolutions. Terms involving b seen to be the simplest. For specific functions $A, B$, and $C$, it is often easier to find the transforas a, $b$, and $c$ and then invert $(4.7),(4.8)$, or (4.9) after the substitutions a nethod for the evaluation of the convolutions, rather than attempting to evaluate the convolutions directly.

ACKNOWLEDGEMNT. Appreciation is expressed to the Department of Mathematics at Oregon State University for providing pleasant working space during sabbatical leave from the UnIversity of Wyoming.

$$
\begin{aligned}
& \text { APPENDIX } \\
& c^{-2}\left\{\frac{1}{8\left(u^{1 / 2}+\mu\right)\left(u^{1 / 2}+8+v\right)}\right\}= \\
& =\frac{1}{v-\mu}\left(v e^{v^{2 x}}\left(\operatorname{Erfc}\left(v x^{1 / 2}\right)-\operatorname{Erfc}\left(\frac{t}{2 x^{1 / 2}}+v x^{1 / 2}\right)\right)-\right. \\
& -\mu e^{\mu^{2} x}\left(\operatorname{Erfc}\left(\mu x^{1 / 2}\right)-e^{(\mu-\nu) t} \operatorname{Brfc}\left(\frac{t}{2 x^{1 / 2}}+\mu x^{1 / 2}\right)\right) \text {. } \\
& c^{-2}\left\{\frac{1}{\left(u^{1 / 2}+\mu\right)\left(u^{1 / 2}+8+v\right)}\right\}=e^{-v t} x(t, x)-e^{(\mu-v) t+\mu^{2} x} \operatorname{Erfc}\left(\frac{t}{2 x^{1 / 2}}+\mu x^{1 / 2}\right) \text {. } \\
& c^{-1}\left\{\frac{\exp \left(-y u^{1 / 2}\right)}{\left(u^{1 / 2}+\mu\right)\left(u^{1 / 2}+\nu\right)}\right\}=\frac{e^{-y^{2} / 4 x}}{\mu-\nu}\left\{\mu E\left(\frac{y}{2 x^{1 / 2}}+\mu x^{1 / 2}\right)-v E\left(\frac{y}{2 x^{1 / 2}}+v x^{1 / 2}\right)\right) \text {. } \\
& c^{-1}\left\{\frac{\exp \left(-y u^{1 / 2}\right)}{\left(u-\mu^{2}\right)\left(u^{1 / 2}+v\right)}\right\}=\frac{e^{-y^{2} / 4 x}}{v^{2}-u^{2}}\left\{\frac{v+\mu}{2} E\left(\frac{y}{2 x^{1 / 2}}+\mu x^{1 / 2}\right)+\right. \\
& \left.+\frac{v-\mu}{2} E\left(\frac{y}{2 x^{1 / 2}}-\mu x^{1 / 2}\right)-v E\left(\frac{y}{2 x^{1 / 2}}+v x^{1 / 2}\right)\right) \text {. }
\end{aligned}
$$

Notation: $\quad E(r)-\exp \left(r^{2}\right) \operatorname{Erfc}(r)$. 


\section{REFERENCES}

1. SUCEC, JAMES, Exact analytical solution to a transient confugate heat-transfer problem, NASA TN D-7101 (1973).

2. SUCEC, JAMES, Unsteady heat transfer between a fluid, with time varying temperature, and a plate: an exact solution, Int. J. Heat Mass Transfer, 18 (1975), 25-36.

3. SINGH, P., SHARMA, V. P., and MISRA, U. N., Exact solution of transient heat transfer between a fluid and a porous plate, Pure App1. Math. Sc1. 10 (1979), 135-144.

4. CARSLAW, H. S. and JABger, J. C., Conduction of heat in Solids, 2nd edition, Clarendon Pres8, Oxford, 1959.

5. VOELKER, D. and DOETSCH, G., Die Zweidimensionale Laplace-Transformation, Verlag B1rkhäuser, Base1, 1950.

6. ROBERTS, G. E. and KAUFMAN, H., Table of Laplace Transforms, W. B. Saunders Co., Phtladelphia, 1966. 


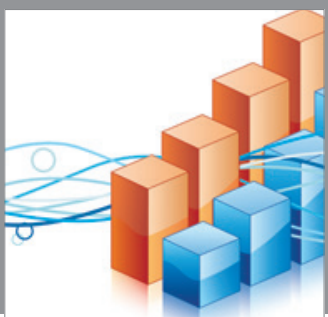

Advances in

Operations Research

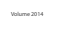

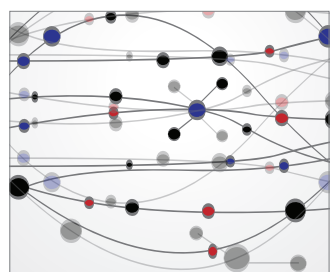

\section{The Scientific} World Journal
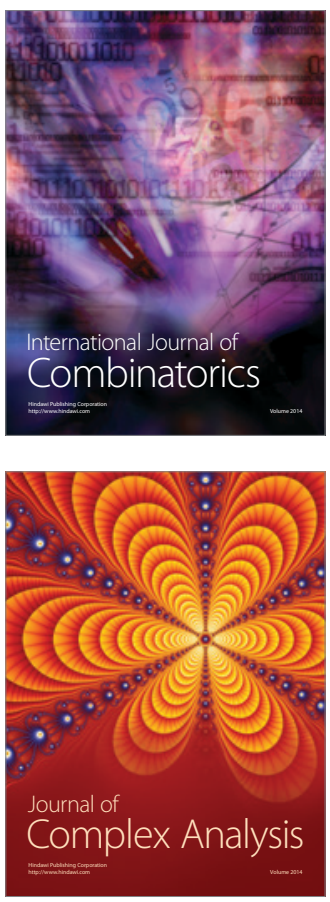

International Journal of

Mathematics and

Mathematical

Sciences
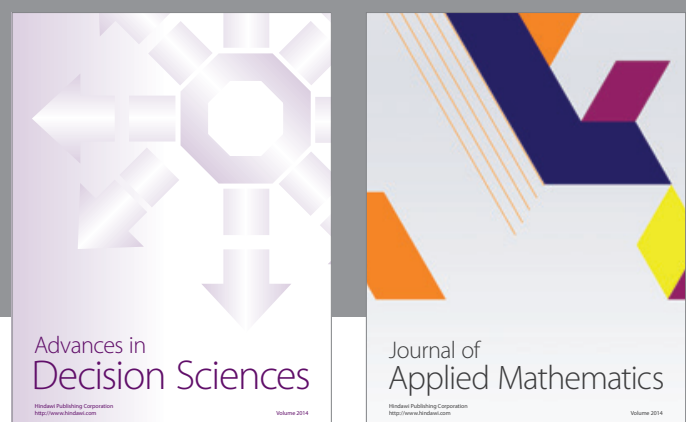

Journal of

Applied Mathematics
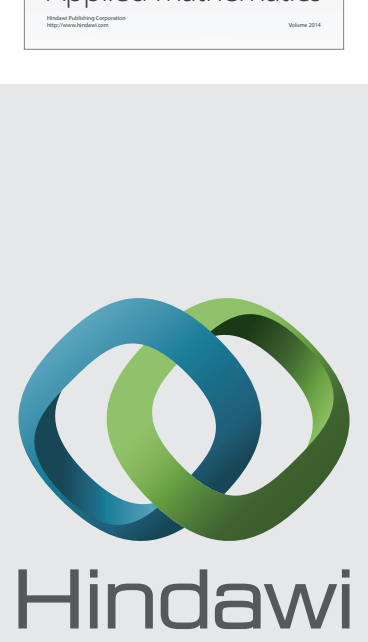

Submit your manuscripts at http://www.hindawi.com
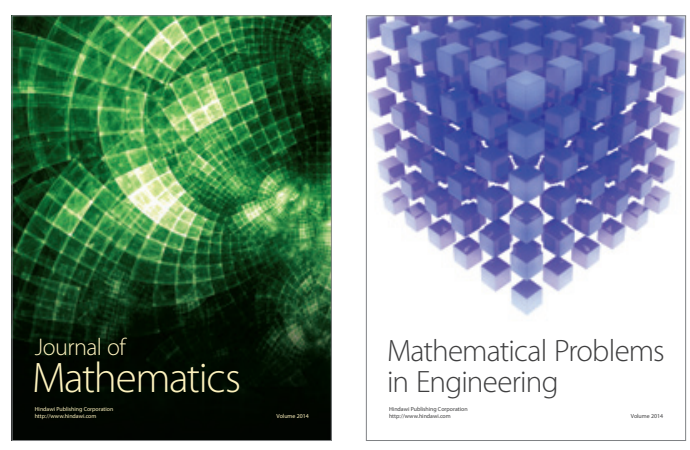

Mathematical Problems in Engineering
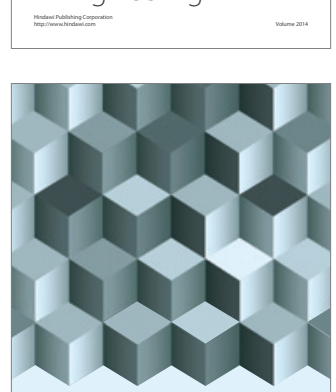

Journal of

Function Spaces
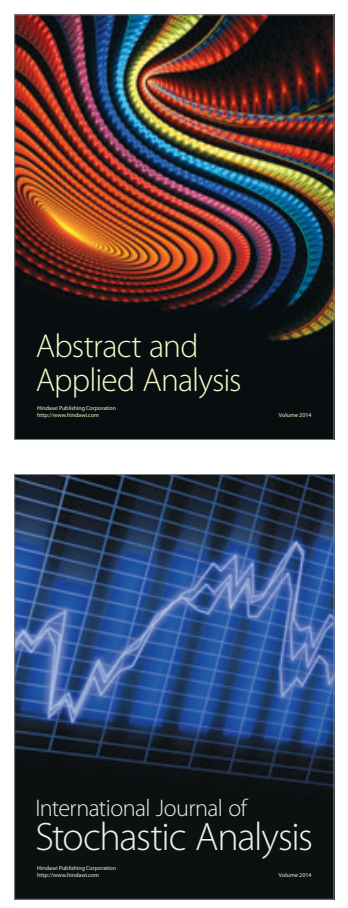

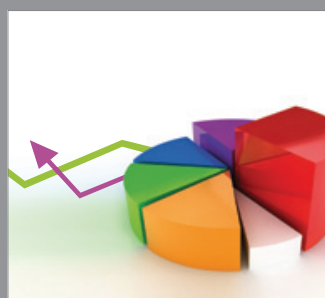

ournal of

Probability and Statistics

Promensencen
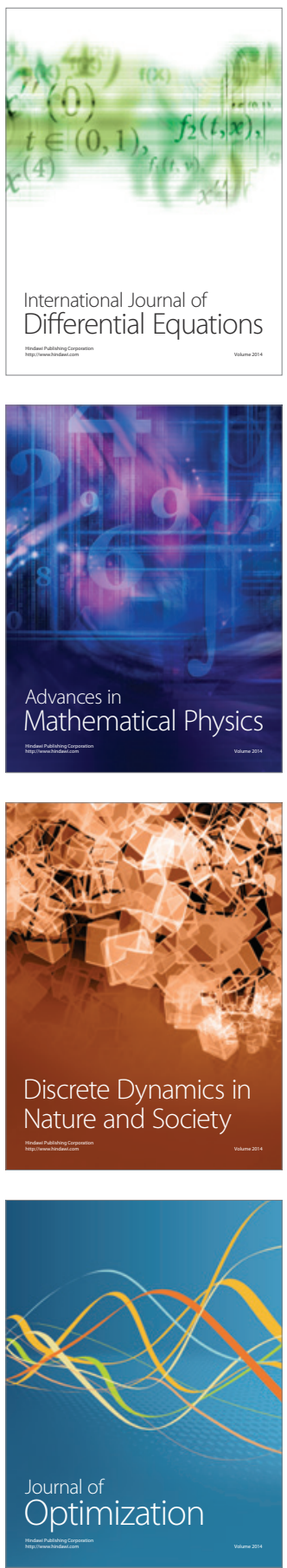